\title{
Enhance System Utilization and Business Revenue with AI-based Queue Reservation System
}

\author{
V. Limlawan and P. Anussornnitisarn
}

\begin{abstract}
Queue management is a crucial part of service industry. Business has to deal with the uncertainty of arrival customer and the trade-off between the costs of providing capacity and customer satisfaction. Matching service capacity with the arrival customer is difficult so the system utilization is low in the low periods and customers have to wait for a long time in the peak period. Due to long waiting, some customer abandons the queue before receiving service. Customer abandonment affects business revenue and the system utilization. To relieve the effect of the customer abandonment, this paper aims to propose Artificial Neural Network based waiting time predictor with queue reservation system. The proposed system increases the system utilization and increase customer satisfaction at the same time. Instead of abandonment, customers can reserve their place in queue if the waiting time is too long and they can do other activities while waiting. Moreover, the proposed system provides the accurate estimated waiting time to each customer instead of queue-length. The accurate waiting time enables arrival customers to better decide on the reservation options. $95 \%$ of our predicted waiting time is accurate within 5 minutes. Comparing to the system without the queue reservation system, the system utilization and the number of the arrival customer completed service improve $13 \%$ and $54 \%$ respectively.
\end{abstract}

Index Terms-Queue management system, waiting time prediction, queue reservation system, artificial neural network.

\section{INTRODUCTION}

Waiting in queue is common in queue daily activities such as buying coffee, seeing a doctor, thus managing queue is a crucial part in service industry. Waiting for a long time in queue has a negative effect on service evaluation [1], [2]. Therefore, many businesses try to reduce the waiting time by providing the service capacity.

Unfortunately, providing too much service capacity increase the operational costs. Moreover, the arrival pattern of customer is random and fluctuate. The management has to deal with the random arrival pattern the trade-off between the costs and customer satisfaction. Thus, the waiting time is too long in peak periods and the system utilization is low in low periods.

In the peak periods, customers have to wait for a long time, and some customer may abandon from the queue before receiving service. The customer abandonment affects

Manuscript received August 15, 2020; revised October 23, 2020.

P. Anussornnitisarn is with the Department of Industrial Engineering, Faculty of Engineering, Kasetsart Univeristy, Thailand (e-mail: fengpta@ku.ac.th)

V. Limlawan is with International Graduated Program in Industrial Engineering, Faculty of Engineering, Kasetsart Univeristy, Thailand (e-mail: varis.lw@gmail.com). business revenue and the system utilization. The service capacity is fixed but the customer received service and the revenue reduce from the abandonment.

Based on the customer abandonment problem, this paper aims to proposed the waiting time predictor based Artificial Neural Network and the queue reservation system which relieve the abandonment of customer. Instead of abandonment, customers are allowed to reserve their place in queue based on the estimated waiting time provided by the predictor.

The remaining of this paper is organized as follows. Section II describes the background of the study. The proposed system is explained in Section III. Experiments and result are discussed in Section IV and Section V respectively. The paper is summarized in Section VI.

\section{BACKGROUND}

To managing queuing system, many businesses, especially bank service, apply Queuing Theory and the computer simulation to reduce the waiting time and to increase the system utilization [3]-[5]. However, the solution from the queuing theory and the computer simulation is optimal when the queuing system reaches the steady-state and must follow the queuing model in the simulation. The queuing system in reality is not stable and does not reach the steady-state because the pattern of arrival customer is random and fluctuate.

Based on the limitation of queuing theory and the simulation, researcher and business focus on managing customer perception. Business generally provide some hospitality such as coffee, WIFI for customers. Furthermore, the methods that is interesting by many researchers is providing time to be served or the estimated waiting time to each customer because customers feel less anxious if they know the time to be served [6].

Many techniques such as the historical based predictor, Queue-length based predictor, machine learning based predictor, artificial neural network is applied to predict the waiting time [7]-[10].

However, no technique can update when the queuing system was changed. To update the system, the artificial neural network-based predictor with the update system is proposed by [11]. Artificial Neural Network (ANN) is selected to be the waiting time predictor because ANN is powerful tool for solving many problems and can handle multi-input and non-linear problems [12]. The system is designed to use the computer vision technology to detect queue-length and the exponential weight moving average (EWMA) to monitor and update the average service time. The 
update queuing parameters are sent to the waiting time predictor.

To provide the estimated waiting time to each customer, most of business recently implement the ticket queue management system. Arrival customers receive a ticket which contain their number and queue information such as queue-length and the estimated waiting time.

Nowadays, the communication technologies such as smartphone has been developed. The ticket queue is also developed by using the technologies and is applied in many places such as hospital, bank, coffee shop [13]-[15]. Customer remotely get the ticket from their smartphone or website.

Although customers are convenient for registering to the queue, the disadvantage of the ticket queue and the developed system is losing queue information [16]. The system does not update the queue-length when customer abandon from the queue the customers often overestimated their waiting time, and lead to the customer abandonment problem

In order to solve the problem, this paper aims to proposed the accurate waiting time predictor and queue reservation system because the reservation can shift the customers from the peak period and the reserved customers is no need to wait for long time [17]. However, the research about the queue reservation has been lack, so this study also proposes the idea of queue reservation which can improve customer satisfaction and the system utilization.

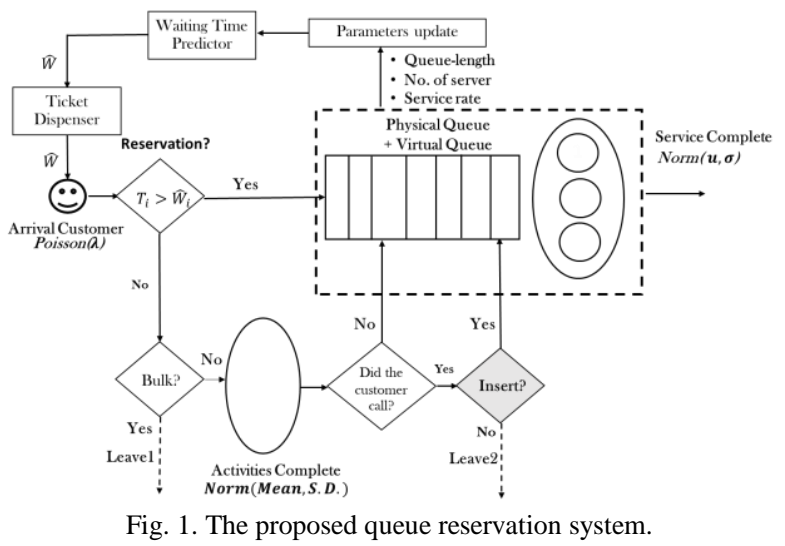

\section{The Proposed QueUe RESERVATION SYSTEM}

The proposed system is the queue management system that allows customers to reserve the queue. As illustrated in Fig. 1, Arrival customers receive the ticket which provide the estimated waiting time. Customers can choose to stay in the waiting area or choose to reserve their place in queue and do other activities before receiving service. For the late reserved customers, customers generally dispose from the queue in the general queue system, but the proposed system has the insert algorithm that insert some customer back to the queue. Customers has a chance to rejoin the queue. The detail of each component will be discussed as follows.

The first component which is important for the system and the customer is the waiting time predictor because customer decide to reserve their queue based on the predicted waiting time. In the proposed system, Artificial Neural Network (ANN) is used as the waiting time predictor, and the inputs of
ANN is queue-length, the number of server and average service time. Based on [11], the average service time is monitored and updated by Exponential Weight Moving Average control chart (EWMA). EWMA is the control chart for detecting the small shift in mean [18].

Let $u$ be the average service time, $\sigma$ be the standard deviation of service time, $b_{i}$ be the estimated service time of customer $i, x_{i}$ be the actual service time of customer $i$, $\lambda$ and $L$ be EWMA parameters. The steps of EWMA are described as follow.

Step 1: Initialize $u, \sigma, \hat{\mathrm{b}}, \lambda$ and $L$

Step 2: Estimate the service time of customer $i$ by using (1)

$$
b_{i}=\lambda x_{i}+(1-\lambda) \hat{\mathrm{b}}_{i-1}
$$

Step 3: Calculate lower bound (LB) and upper bound (UB) as using (2) and (3)

$$
\begin{aligned}
& L B=u-L \sigma \sqrt{\frac{\lambda}{(2-\lambda)}\left[1-(1-\lambda)^{2 i}\right]} \\
& L B=u+L \sigma \sqrt{\frac{\lambda}{(2-\lambda)}\left[1-(1-\lambda)^{2 i}\right]}
\end{aligned}
$$

Step 4: If $\hat{b}_{i}>U B$ or $\hat{b}_{i}<U B$ then $u<U B$

Step 5: Repeat step 2 until the system termination.

Once the queue parameters are updated, the parameters are sent to ANN. The architecture of ANN in the proposed system is a single hidden layer feedforward as shown in Fig. 2.

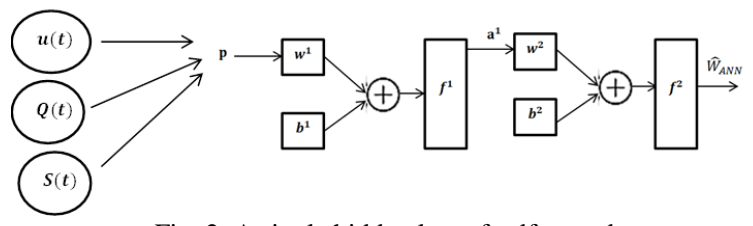

Fig. 2. A single hidden layer feedforward.

Based on [19], the single hidden layer feedforward consists of 3 layers which are input layer, hidden layer and the output layer. There are M hidden neuron in the hidden layer. In this research, the inputs of the ANN are queue-length, number of servers and average service time which is represented as follow.

$$
\mathbf{P}=\left[\begin{array}{c}
u(\mathrm{t}) \\
Q(t) \\
\mathrm{s}(t)
\end{array}\right\rfloor \text {, for } t=1,2,3, \ldots
$$

where $u(t)$ is the average service time at time $t, Q(t)$ is queue-length at time $t, s(t)$ is the number of servers at time $t, t$ is the discrete time, $\mathbf{P}$ is the inputs vectors of ANN.

The output of $i$ layer is the input of $i+1$ layer. The output of the output layer is the predicted waiting time denoted as $\hat{w}_{A N N}$. The output of each layer is calculated by using (5)-(7)

$$
\begin{gathered}
\mathbf{a}^{0}=\mathbf{P} \\
\mathbf{a}^{i+1}=f^{i+1}\left(\mathbf{w}^{i+1} \mathbf{a}^{i}+\mathbf{b}^{i+1}\right) \text { for } i=0,1 \\
\mathbf{a}^{2}=\left[\hat{w}_{A N N}\right]
\end{gathered}
$$


where $\mathbf{a}^{i}$ is the input vector of layer $i, \mathbf{b}^{i}$ is the biased vector of layer $i, \mathbf{w}^{i}$ is the weights of layer $i, f^{i}$ is the activate function of layer $i$.

The predicted waiting time from ANN is provided to each customer. Customers compare the predicted waiting time with the tolerance time. Let $T_{i}$ the tolerance time of customer $i$ and $\hat{w}_{i}$ be the predicted waiting time of customer $i$.

If $T_{i}<\hat{w}_{i}$, the customers will reserve the queue, customers would reserve the queue. Otherwise, the customers will not reserve and stay in the waiting area provided by services. The reserved customers do their activities such as shopping, having lunch and come back to the queue and receive the service.

However, there is some reserved customer who come back and their queue is calling. The late customers generally re-entry to the service at the back of the queue, but the proposed system has a special feature called "insert algorithm" which considers and inserts the late customers to the queue. The reserved customers who are late have a chance to rejoin the queue.

The idea of the insert algorithm is searching the available time slot in queue. The available time slot of customer $i$ is calculated by (8)

$$
D_{i}=\hat{w}_{i}-E_{i}-\hat{R}_{i}
$$

where $\hat{w}_{i}$ is the estimated waiting time, $E_{i}$ is the elapsed time from the arrival time, $\hat{R}_{i}$ is the remaining waiting time calculated from the position of customer $i, D_{i}$ is the available time slot of customer $i$ in queue. As shown in Fig. 3, the steps of the insert algorithm are described as follows.

Step 1: $\quad$ Select customer $k$

Step 2: Check queue-length, denoted as $N Q$

If $N Q>0$, then set $i=1$. Otherwise insert customer to the queue.

Step 3: Calculate the available time slot of customer $i$

Step 4: If $D_{i}>1$, insert customer $k$ to $i$ position of the queue. Otherwise, go to step 5.

Step 5: If $i=N Q$, dispose customer $k$ to $i$ position of the queue. Otherwise, set $i=i+1$ and repeat step 3 .

\section{EXPERIMENT}

To evaluate the proposed system with the reservation system, the experiments are designed to compare the proposed queue reservation system and the queue system without the queue reservation. The experiment setting is described as followed.

\section{A. Simulation of the Test System}

The test system is dynamic multi-server queue systems, and are simulated by SIMAN ARENA® Rockwell software. The detail of the system is described as followed.

The system opens at 8.00 a.m. and closes at 16.00 p.m. The Service time is normal distribution with a mean of 1 and a standard deviation of 0.75 . The system load (p) and number of servers are dynamically changed based on the schedule in
Table I, and the arrival rate is Poisson distribution with a mean of $\lambda$ which is adjusted based on the load $\rho$, and number of servers.

The simulation of the proposed queue reservation system is built based on the diagram in Fig. 1. Customers decide to reserve or stay based on the waiting time and their tolerance time. The reserved customer does other activities and return to the queue. The reserved customer who their queue has been called is considered to rejoin the queue by the insert algorithm.

For the queuing system without queue reservation system, the simulation of the system is built as shown in Fig. 4. Customer decide to abandon or stay based on the waiting time and their tolerance time. The abandoned customer also does other activities and return to the queue, but the customer who their queue has been called must be disposed from the queue or rejoin the queue at the back of the queue.

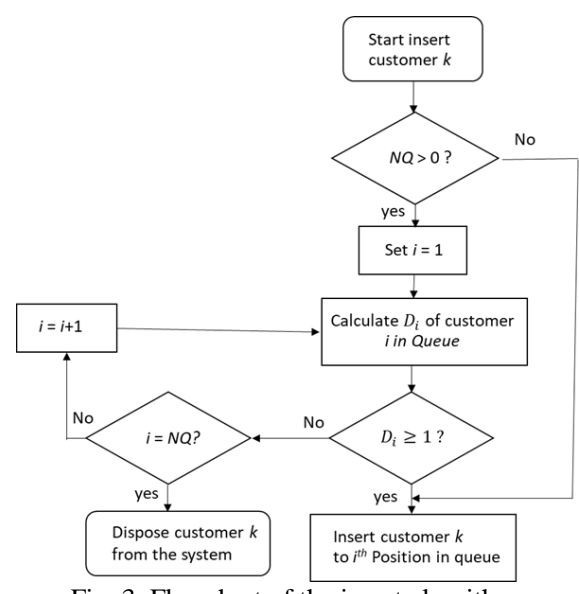

Fig. 3. Flowchart of the insert algorithm.

TABLE I: THE SCHEDULE OF THE TEST SYSTEM

\begin{tabular}{|c|c|c|c|c|}
\hline Time & $\begin{array}{c}\text { Arrival Rate }(\lambda) \\
\text { (min/customer) }\end{array}$ & Service Time & Resource & P(Load) \\
\hline $8.00-9.00$ & 1.25 & Norm $(1,0.75)$ & 1 & 0.80 \\
\hline $9.00-10.00$ & 1.05 & Norm $(1,0.75)$ & 1 & 0.95 \\
\hline $10.00-11.00$ & 0.51 & Norm $(1,0.75)$ & 2 & 0.99 \\
\hline $11.00-12.00$ & 0.45 & Norm $(1,0.75)$ & 2 & 1.1 \\
\hline $12.00-13.00$ & 0.30 & Norm $(1,0.75)$ & 3 & 1.1 \\
\hline $13.00-14.00$ & 0.51 & Norm $(1,0.75)$ & 2 & 0.99 \\
\hline $14.00-15.00$ & 1.05 & Norm $(1,0.75)$ & 1 & 0.95 \\
\hline $15.00-16.00$ & 1.25 & Norm $(1,0.75)$ & 1 & 0.80 \\
\hline
\end{tabular}

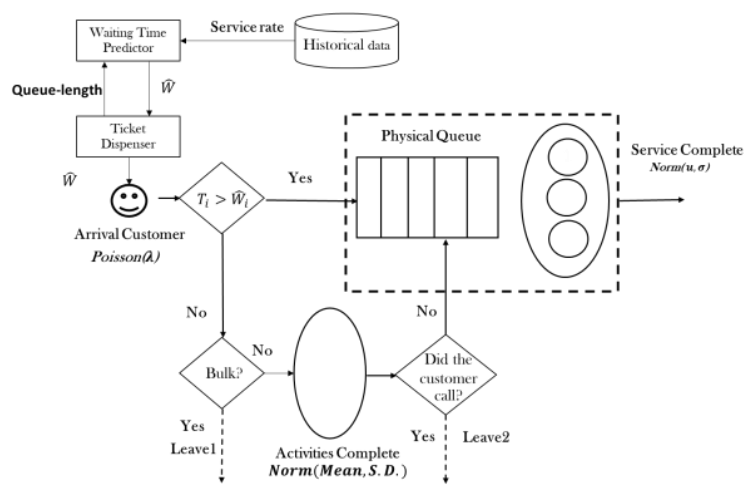

Fig. 4. Diagram of the system without queue reservation system.

\section{B. Test Case}

In order to evaluated the proposed system, the experiment is designed based on the customer abandonment as shown in 
Table II. There are three levels of customer abandonment which are low, medium and high. The abandonment level is varied by adjusting the tolerance time. The tolerance time of customer is exponential distribution with mean of $\gamma$.

The activity time outside the shop of each customer is normal distribution with mean $u_{t}$ and $\sigma_{t} \cdot u_{t}$ is calculated based on the estimated waiting time of each customer, denoted as $\hat{w}_{i}$, and $\sigma_{t}$ is equal to $0.5 u_{t}$

For all cases, the other queue parameters are set based on the schedule. Training set, validation set and test set are run 30,10 and 10 respectively.

\section{Parameter Setting}

ANN is programmed by MATLAB R2016a. The number of neurons in the hidden layer is 5 . The activated function of the hidden layer is tan-sigmoid function and the activated function of the output is pure linear function. ANN is trained by backpropagation algorithm which the learning rate is 0.05 . $\lambda$ and L of EWMA control chart in the monitoring system are 0.1 and 2.7 respectively. ANN is trained by 30 replications of the test case.

\section{Performance Measurement}

The objective of this study is to proposed the system with reservation feature that can reduce customer abandonment which affects the business revenue and the operation efficiency. Percentage of customer completed service and the utilization of the system are used to evaluate the system performance. The utilization of the system is generated by SIMAN ARENA® Rockwell software [20]. Let $P C$ is the percentage of customer completed service. The percentage of customer completed service is calculated by using (9).

$$
P C=\frac{1}{N} \sum_{i=1}^{N} r_{i} \times 100
$$

where $r_{i}= \begin{cases}1 & , \text { customer completed service } \\ 0 & , \text { otherwise }\end{cases}$

where $N$ is the number of arrival customers, $r_{i}$ is the binary variable which indicate that which customers completed the service.

In order to compare the proposed system and the system without queue reservation, the percentage of improvement from the system without queue reservation is used as the performance measurement. The percentage of improvement is denoted as \%improvement and calculated by (10).

$$
\text { \%improvement }=\frac{(\mathrm{P}-O)}{O} \times 100
$$

where $P$ is the performance of the proposed system and $O$ is the performance of the system without queue reservation.

The accuracy of the waiting time predictor needs to be evaluated since the predicted waiting time is important for all types of customers. The reserved customer plans their activities based on the waiting time prediction. The error of waiting time prediction is calculated by (11).

$$
e_{i}=w_{i}-\hat{w}_{i}
$$

where $e_{i}$ is error of the waiting time prediction of customer $i$, $w_{i}$ is the actual waiting time of customer $i, \hat{w}_{i}$ is the predicted waiting time of customer $i$.

The waiting time prediction for all customers is evaluated by percentage of accuracy within the acceptance tolerance such as 1 minutes, 3 minutes. Let $N$ be the number of arrival customers, $c_{i}$ be the accuracy indication of customer $i, l$ be the acceptance tolerance and $\% P A$ within $l$ be the percent accuracy within $\pm l$. The percentage of the accuracy within $l$ is calculated by (12) and (13)

$$
\begin{gathered}
c_{i}= \begin{cases}1 & , e_{i} \in[-l, l] \\
0 & , \text { otherwise }\end{cases} \\
\% P A \text { within } l=\frac{1}{N} \sum_{i=1}^{N} c_{i} \times 100
\end{gathered}
$$

\section{RESUlT AND DiSCUSSION}

To check the model, the customer information such as the customer abandonment, the reserved customer is presented in Table III. In the system without queue reservation, the percentage of reservation in low abandonment, medium abandonment and high abandonment is $8.41 \%, 33.94 \%$ and $60.47 \%$ respectively.

In the proposed system, the percentage of abandonment in low abandonment, medium abandonment and high abandonment is $16.79 \%, 58.81 \%$ and $80.41 \%$ respectively. And, the percentage of late reserved customer in low abandonment, medium abandonment and high abandonment is $19.54 \%, 23.37 \%$ and $29.12 \%$ respectively.

Based on the result, the simulation model is corrected. The percentage of reservation and the percentage of abandonment increases as the tolerance time decreases. The percentage of late reserved customer slightly increase as the tolerance time decrease. In addition, the percentage of reservation in the proposed system is more than the percentage of the abandonment in the system without reservation because the queue reservation increases the load of the system.

The accuracy of ANN based predictor in the proposed system is reported in Table IV. The result shows that more than $99 \%$ of the predicted waiting time is accurate within 10 minutes and more than $95 \%$ of the predicted waiting time is

\begin{tabular}{|c|c|c|c|}
\hline \multirow[b]{2}{*}{ Case } & $\%$ Abandonment & $\%$ Reservation & \multirow{2}{*}{$\begin{array}{l}\% \text { Late of } \\
\text { Reserved } \\
\text { Customer }\end{array}$} \\
\hline & $\begin{array}{c}\text { W/o } \\
\text { Reservation }\end{array}$ & $\begin{array}{c}\text { With } \\
\text { Reservation }\end{array}$ & \\
\hline $\begin{array}{l}\text { High } \\
\text { Abandonment }\end{array}$ & $60.47 \%$ & $80.41 \%$ & $29.12 \%$ \\
\hline $\begin{array}{l}\text { Medium } \\
\text { Abandonment }\end{array}$ & $33.94 \%$ & $58.81 \%$ & $23.37 \%$ \\
\hline $\begin{array}{l}\text { Low } \\
\text { Abandonment }\end{array}$ & $8.41 \%$ & $16.79 \%$ & $19.54 \%$ \\
\hline
\end{tabular}
accurate within 5 minutes.

TABLE II: TEST CASE

\begin{tabular}{|c|c|c|c|}
\hline Case & $\begin{array}{c}\text { Tolerance } \\
\text { Time }\end{array}$ & $\begin{array}{c}\text { Activity Time } \\
\text { while waiting }\end{array}$ & $\begin{array}{c}\text { Queue } \\
\text { System } \\
\text { Parameters }\end{array}$ \\
\hline Low Abandonment & $\operatorname{Expo(30)}$ & Norm & $\begin{array}{c}\text { Based on } \\
\text { the } \\
\text { Schedule }\end{array}$ \\
\cline { 1 - 2 } Medium Abandonment & $\operatorname{Expo}(5)$ & $\left(\hat{w}_{i}-\sigma_{t}, 0.5 \mu_{t}\right)$ & $\operatorname{Expo}(1)$
\end{tabular}

TABLE III: CUSTOMER INFORMATION 
TABLE IV: PERCENTAGE OF ACCURACY OF ANN BASED PREDICTOR

\begin{tabular}{|l|c|c|c|c|}
\hline \multicolumn{1}{|c|}{ Case } & $\begin{array}{c}\% \mathrm{PA} \\
\text { within 1 } \\
\text { min }\end{array}$ & $\begin{array}{c}\text { \%PA } \\
\text { within 3 } \\
\text { min }\end{array}$ & $\begin{array}{c}\% \mathrm{PA} \\
\text { within 5 } \\
\text { min }\end{array}$ & $\begin{array}{c}\% \mathrm{PA} \\
\text { within 10 } \\
\text { min }\end{array}$ \\
\hline High Reservation & $55.1 \%$ & $91.4 \%$ & $97.7 \%$ & $99.9 \%$ \\
\hline $\begin{array}{l}\text { Medium } \\
\text { Reservation }\end{array}$ & $50.8 \%$ & $87.8 \%$ & $95.7 \%$ & $99.4 \%$ \\
\hline Low Reservation & $57.0 \%$ & $92.6 \%$ & $98.2 \%$ & $99.9 \%$ \\
\hline
\end{tabular}

Based on the result, ANN in the proposed system can provide the accurate waiting time to each customer. The accurate waiting time enables arrival customer to better decide on the reservation option; moreover, the accurate time to be serve reduce the anxiety of customers who waits at the waiting area.

The comparison between the proposed system and the system without queue reservation system are presented in Table V, Table VI, Fig. 5 and Fig. 6. The proposed system outperforms the system without queue reservation. Implementing the proposed system results in improvement of $54 \%, 28 \%$ and $7 \%$ in high abandonment case, medium abandonment case and low abandonment case respectively. And, the utilization of the system with the proposed system improves $13 \%, 6 \%$ and 5\% in high abandonment case, medium abandonment case and low abandonment case respectively.

Based on the result, the proposed system outperforms the system without queue reservation in all cases. With the queue reservation system, business can gain more revenue and increase the utilization of the system.

TABLE V: COMPARISON OF THE PERCENTAGE OF CUSTOMER COMPLETED

\begin{tabular}{|c|c|c|c|}
\hline \multicolumn{4}{|c|}{ SERVICE } \\
\hline Case & $\begin{array}{c}\text { W/o } \\
\text { Reservation }\end{array}$ & $\begin{array}{c}\text { With } \\
\text { Reservation }\end{array}$ & $\begin{array}{c}\% \\
\text { Improvement }\end{array}$ \\
\hline $\begin{array}{l}\text { High } \\
\text { Abandonment }\end{array}$ & $57.75 \%$ & $89.16 \%$ & $54 \%$ \\
\hline $\begin{array}{l}\text { Medium } \\
\text { Abandonment }\end{array}$ & $72.69 \%$ & $93.16 \%$ & $28 \%$ \\
\hline $\begin{array}{l}\text { Low } \\
\text { Abandonment }\end{array}$ & $91.61 \%$ & $97.86 \%$ & $7 \%$ \\
\hline
\end{tabular}

TABLE VI: COMPARISON OF THE SYSTEM UTILIZATION

\begin{tabular}{|l|c|c|c|}
\hline \multicolumn{1}{|c|}{ Case } & $\begin{array}{c}\text { W/o } \\
\text { Reservation }\end{array}$ & $\begin{array}{c}\text { With } \\
\text { Reservation }\end{array}$ & $\begin{array}{c}\% \\
\text { Improvement }\end{array}$ \\
\hline $\begin{array}{l}\text { High } \\
\text { Abandonment }\end{array}$ & 0.78 & 0.88 & $13 \%$ \\
\hline $\begin{array}{l}\text { Medium } \\
\text { Abandonment }\end{array}$ & 0.88 & 0.93 & $6 \%$ \\
\hline $\begin{array}{l}\text { Low } \\
\text { Abandonment }\end{array}$ & 0.89 & 0.93 & $5 \%$ \\
\hline
\end{tabular}

\% Completed Service of the arrival customer

- Without Reservation $\quad$ With Reservation

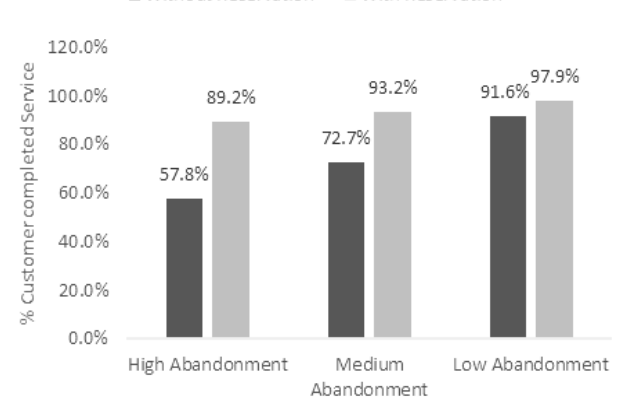

Fig. 5. Plot of percentage completed service of both systems.

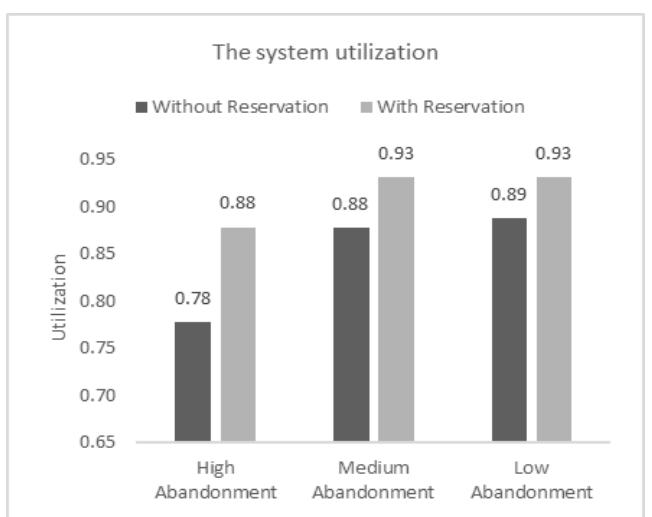

Fig. 6. Plot of the system utilization of both systems.

\section{CONCLUSION}

In this paper, we proposed the queue reservation system with artificial neural network-based waiting time predictor. The customer abandonment problem affects the business revenue and the system utilization, so the proposed system is developed for alleviating the customer abandonment problem.

The proposed system allows customer to reserve their place in queue if the waiting time is too long. The system provides the predicted waiting time each customer by using Artificial Neural Network. Unlike other systems, the proposed system updates the inputs of the waiting time predictor. Exponential Weight Moving Average (EWMA) control chart is applied to monitor and update the average service time.

Customers decide to reserve the queue based on the provided waiting time, and do other activities such as having dinner, shopping while waiting. However, some customers return to the shop and their queue has been called. The late customers are generally disposed from the queue and have to rejoin the queue at the back of the queue or abandon the queue. In the proposed system, the customers have a chance to rejoin the queue by the insert algorithm.

The proposed system is compared with the system without the queue reservation system. The result shows that the proposed system can increase business revenue and increase the utilization of the system.

For further study, the lateness of the reserved customers should be studied because the lateness of the reserved customers is random. Moreover, the communication technology such as smartphone should be implemented with the queue reservation system. The technology will allow customers to join or reserve their queue via their smartphone and can also check their queue status in real-time.

\section{CONFLICT OF INTEREST}

The authors declare no conflict of interest.

\section{AUTHOR CONTRIBUTIONS}

P. Anussornnitisarn and V. Limlawan devised the main conceptual ideas. P. Anussornnitisarn provided the technical support and the direction for this research. V. Limlawan. worked out the technical details, and performed the numerical calculations for the suggested experiment. P. Anussornnitisarn verified and approved the experiments, and 
supervised the findings of this work. P. Anussornnitisarn and V. Limlawan wrote the paper. All authors had approved the final version.

\section{REFERENCES}

[1] K. L. Katz, B. M. Larson, and R. C. Larson, "Prescription for the waiting-in-line blues: Entertain, enlighten, and engage," Sloan Management Review, vol. 32, no. 2, pp. 44-53, 1991.

[2] S. Tarloy, "Waiting for service: The relationship between delays and evaluations of service," Journal of Marketing, vol 52, no. 1, pp. 56-69, 1994.

[3] A. AL-Jobori, D. H-J. AL-Jumaily, "Automatic queuing model for banking applications," International Journal of Advanced Computer Science and Applications (IJACSA), vol. 2, no. 7, pp. 11-15, 2011.

[4] E. Berhan, "Bank service performance improvements using multi-sever queue system," Journal of Business and Management, vol. 17, pp. 65-69, 2015.

[5] B. Patel and P. Bhathawala, "Case study for bank ATM Queuing model," International Journal of Engineering Research and Application (IJERA), vol. 2, no. 5, pp. 1278-1284, 2012.

[6] R. Ibrahim and W. Whitt, "Real-time delay estimation based on delay history," Manufacturing \& Service Operations Management Manufacturing \& Service Operations Management, vol. 11, no. 3, pp. 397-415, 2009.

[7] A. Senderovich, M. Weidlich, and A. Mandelbaum, "Queue mining predicting delays in service processes," International Proceedings of Advanced Information Systems Engineering, pp. 42-57, 2014.

[8] Y. Sanit-in, K. Saikaew, "Prediction of waiting time in one-stop service," International Journal of Machine Learning and Computing, vol. 9, no. 2, pp. 322-327, 2019.

[9] D. Maister, "The psyhchology of waiting lines," in The Service Encounter: Managing Employee/Customer Interaction in Service Businesses, J. A. Czepiel, M. R. Solomon, and C. F. Surprenant, Eds., Lexington, MA: D. C. Heath and Company, Lexington Books, 1985.

[10] M. Thiongane, W. Chan and P. L. Ecuyer, "Waiting time predictors for multi-skill call centers," in Proc. the 2015 Winter Simulation Conference, Huntington Beach, CA, USA, 2015, pp. 3073-3084.

[11] V. Limlawan and P. Anussornnitisarn, "Development of waiting time predictor based Artificial Neural Network," in Proc. IOP Conference Series: Materials Science and Engineering, vol. 847, 2020.

[12] O. I. Abiodun, A. Jantan, A. E. Omolara, K. V. Dada, and N. A. Mohamed, "State-of-the-art in artificial neural network applications: A survey," Heliyon, vol. 4, no. 11, 2018.

[13] D. Uhler, K. Mehta, J. Wong, M. Bulut, Y. Yilmaz, M. Demirbas, and H. Ferhatosmanoglu, "LineKing: Crowdsourced line wait-time estimation using smartphones," Mobile Computing, Applications, and Services, vol. 10, pp. 205-224, 2015.
[14] M. Ngorsed and P. Sueaowaluk, "Hospital service queue management system with wireless approach," in Proc. the 4th International Conference on Frontier Computing, 2015, pp. 627-637,vol. 375.

[15] M. Ghazal, R. Hamouda, and S. Ali, "An IoT smart queue management system with real-time queue tracking," in Proc. 2015 Fifth International Conference on e-Learning, 2015, pp. 257-262.

[16] S. H. Xu, L. Gao, and J. Ou, "Service performance analysis and improvement for a ticket queue with balking customers," Management Science, vol. 53, no. 6, pp. 971-990, 2007.

[17] M. E. McGinley, X. Zhu, and M. Veeraraghavan, "On reservation systems and queueing systems," presented at the 2014 4th IEEE International Conference on Information Science and Technology, Shenzhen, China, April 26-28, 2014.

[18] D. C. Montgomery, Introduction to Statistical Quality Control, 7th ed, John Wiley \& Sons, Inc., 2012, ch. 8, pp. 405-416.

[19] M. T. Hegan, H. B. Demuth, M. H. Beale, and O. D. Jess. (November 2019). Neural Network Design. [Online]. Available: https://hagan.okstate.edu/NNDesign.pdf

[20] W. D. Kelton, R. Sadowski, and N. Zupick, Simulation with Arena, 6th ed, Boston: McGraw-Hill, 2015.

Copyright $\odot 2021$ by the authors. This is an open access article distributed under the Creative Commons Attribution License which permits unrestricted use, distribution, and reproduction in any medium, provided the original work is properly cited (CC BY 4.0).

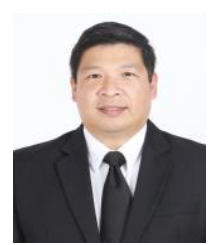

Pornthep Anussornnitisarn is an assistant professor and head of the Department of Industrial Engineering at Kasetsart University Thailand. He received his doctora degree in industrial engineering from the Purdue University in 2003. His research interests include manufacturing execution systems and multi - agent based decision-making systems, asynchronous and stochastic simulations, applications of metaheuristics and artificial intelligence in manufacturing environment, applications of parallel algorithm

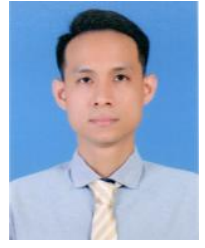

Varis Limlawan is a doctoral student in industrial engineering at International Graduate Program in Industrial Engineering, Faculty of Engineering, Kasetsart University Thailand. His research interests include operation research, queuing system, logistics and supply chain system. 